\title{
Performance analysis of first iteration koch curve fractal log periodic antenna of varying flare angles
}

\author{
Ashutosh K. Singh ${ }^{1 *}$, Reneez A. Kabeer ${ }^{1}$, Z. Ali ${ }^{2}$, V.K. Singh ${ }^{2}$, M. Shukla ${ }^{3}$ \\ 1 Department of Electronics Engineering, Indian Institute of Information Technology, Allahabad, India \\ 2 Department of Electronics Engineering, Bundelkhand University, Jhansi, India \\ 3 Department of Electronics Engineering, Harcourt Butler Technological Institute, Kanpur, India
}

Received 07 August 2012; accepted 08 September 2012

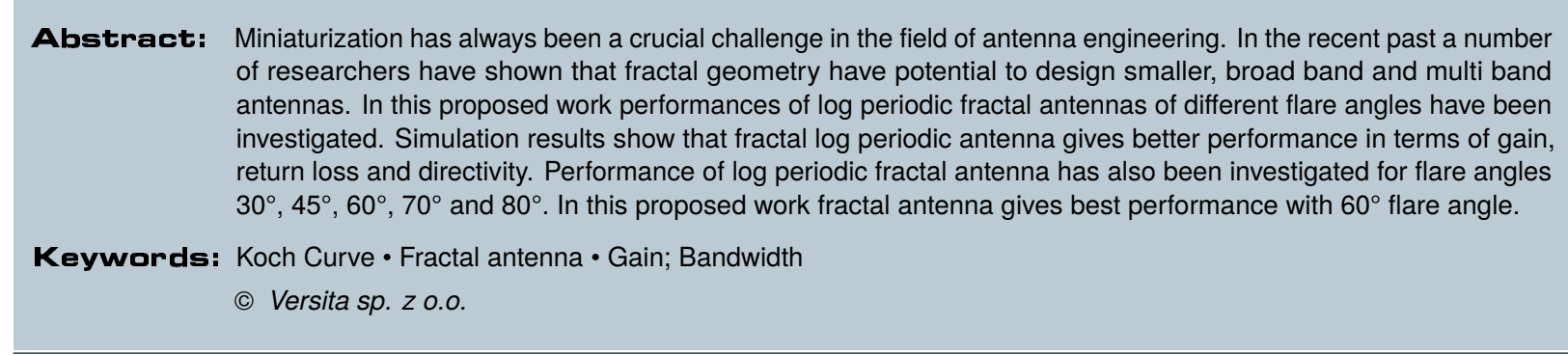

\section{Introduction}

In the field of wireless communication, antenna size and space occupied by it are very crucial factors. The antenna has to be compact as well as feasible, as long as fabrication is concerned, along with having an improvement or a nonconsiderable degradation in its radiation patterns and performance. This is where fractal geometry comes into the picture with fractal antennas.

A fractal is a fragmented geometric shape which is made of reduced-size copies of itself. In other words, fractals are self-similar structures or shapes. Fractal antennas are those antennas which use this self-similarity property of

*E-mail: ashutosh_singh@iiita.ac.in fractals on each of its elements to form a fractal antenna. The first of its kind was the log periodic antenna. These fractal antennas are composed of many reduced-size copies of itself in it and hence provide a wider range of operational frequencies. The general rule of antenna length being near target frequency wavelength does not apply itself in the same way with fractal antennas. Although effective electrical length of the elements of fractal antenna is longer, the fractal antennas are themselves physically smaller. Thus these fractal antennas, due to their compact size and multi-band properties, are very useful in cellular telephone and microwave communications.

There are various types of fractal structures and geometrical shapes introduced. Some of the examples are Koch Curve, Serpinski triangle, T-Square, Gosper Island, etc. The Koch curve is one of the earliest mathematical curves described according to fractal geometry and can have infi- 


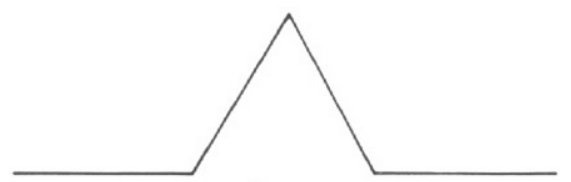

Stage 1

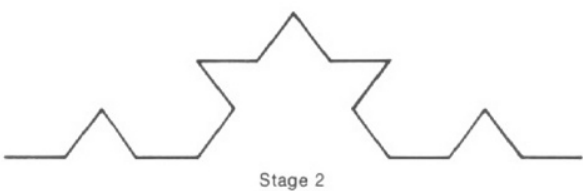

Stage 2
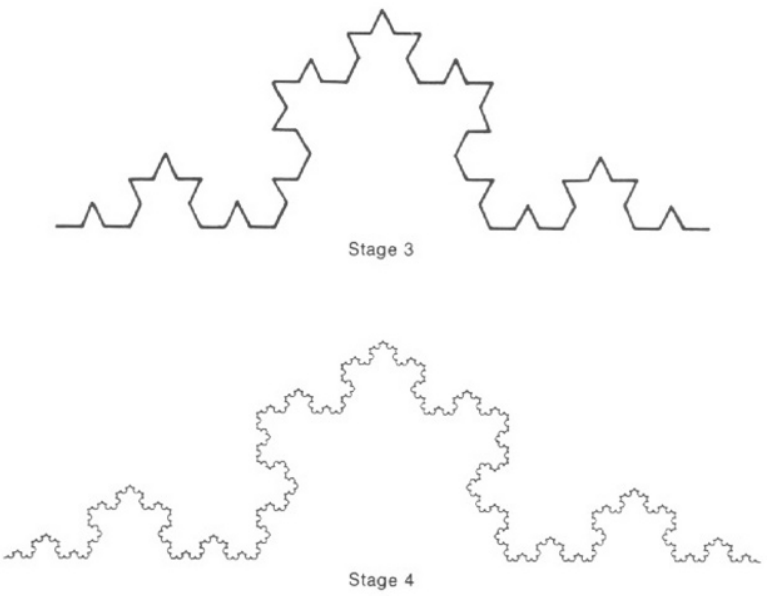

Adapted from Benoit Mandelbrot, Fractals.

Figure 1. Koch curve fractal and its 4 iterations.

nite number of iterations. The Koch "fractalization" is done to an element by dividing the element into three equal parts ' $a, b, c$ ' of length ' $l$ ' and replace the center element 'b' with two elements joint together at a flare angle $60^{\circ}$, as in Figure 1.

The log periodic Koch fractal antenna uses the above mentioned Koch curve fractal to modify the various elements of the conventional log periodic dipole antenna. The different iterations of these antennas can be denoted as $\mathrm{K} 0$ and $\mathrm{K} 1$ being $0^{\text {th }}$ and $1^{\text {st }}$ iterations. In the $1^{\text {st }}$ iteration, the angle at which the center two elements meet is called the "flare" angle. This flare angle can be of any magnitude, but conventionally is maintained at $60^{\circ}$ to maintain a constant overall length of element. The conventional log periodic antenna can also be called the $0^{\text {th }}$ iteration log periodic Koch fractal antenna. The log periodic Koch fractal antenna is shown to be more compact than the conventional log periodic antenna while maintaining its overall performance characteristics with minimal degradation in input impedance and return loss [1]

\section{Related work}

Most of the research work in antenna designing is done improving the performance of the antenna keeping size similar or miniaturizing the antenna with very less difference in performance. In [1] the authors have used Koch curve fractal over a conventional Log Periodic dipole antenna to show the difference in performance. This was the first application of Koch pre-fractal elements in a miniaturized wideband antenna design. In [2], the concept of fractal antenna was employed to achieve the necessary height reduction, while keeping its radiation characteristics nearly constant. Research paper [3] deals with a type of fractal shape known as Serpinski triangle and its use on a monopole antenna.

The PKG (Penta-Gasket-Koch) fractal has been implemented to design a planar monopole antenna with satisfying input impedance match and gain in [4]. In the paper "Fractal log-periodic Antenna" [5], the authors have discussed the effect of Koch fractal on the conventional log periodic antenna along with simulated patterns and results. They describe how each of these iterations causes an increase in the electrical length of the antenna while keeping the size of the antenna almost constant. And also how the fractals affect the input impedance and return loss parameters.

In the field of antenna design, the topic of fractal geometry and fractal designs have recently gained a considerable amount of interest. One such example is the paper [6], in which, an improvement has been made to the fractal tree structure applied to a log periodic antenna, published in paper [7]. Yet another example of the advantages of fractal geometries and fractal structures on dipoles has been studied in the paper [8]. Log periodic antenna is very widely used in VHF/UHF range for commercial purposes. Therefore its size reduction was very crucial step in antenna designing field as shown in paper [9].

In 1996, D.H. Werner, P.L. Werner and A.J Ferraro discussed the frequency independent properties of fractal structures [10] or structures which possessed the selfsimilarity property. The paper has shown how these fractal shapes and geometrical structures can be used to develop multi band linear array radiators whose directive gain is a $\log$ periodic function of frequency. This antenna is the one commonly used today as the log periodic dipole array antenna (LPDA). In 1997, an author, N. Cohen, [11] published an article on the applications of fractal geometry to antenna elements for smaller, resonant multiband/broadband antennas which are simple and cost-effective to fabricate. In this paper, N. Cohen has also explained how these fractal shapes on antennas can help in the miniaturization of size and provide better resonance. The paper also shows 


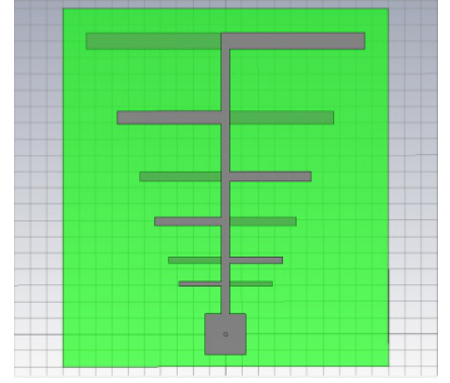

Figure 2. LPKFA with flare angle $0^{\circ}$.

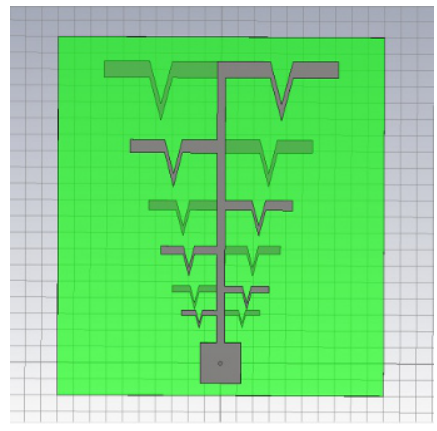

Figure 3. LPKFA with flare angle $30^{\circ}$.

the multi-banded nature of such fractal antennas and the expansion in bandwidths.

In paper [12], the authors have designed different iterations of Koch dipole log periodic antenna for size reduction. Recently work was done on a multi-fractal antenna combining the Koch fractal and the Serpinski fractal for application over a dipole antenna [13].

\section{Proposed work}

In this paper, the computer software CST - Microwave Studio ${ }^{\circledR}$ is used to design and simulate a Log Periodic Koch fractal antenna of $1^{\text {st }}$ iteration. First, a conventional Log Periodic antenna is designed or a $0^{\text {th }}$ iteration Log Periodic Koch fractal antenna. Then, the $1^{\text {st }}$ iteration antenna is going to be designed by applying Koch fractal to the conventional antenna. The designs are going to be for different "flare" angles namely at $30^{\circ}, 45^{\circ}, 60^{\circ}, 70^{\circ}$ and $80^{\circ}$.

Finally, all the designs are simulated in the simulation software to get the required antenna performance parameters. These results for different flare angles are compared to provide a conclusion to this paper.

The above figures (Figure 2 to Figure 7) are of the designed $\log$ periodic Koch $1^{\text {st }}$ iteration fractal antenna with flare

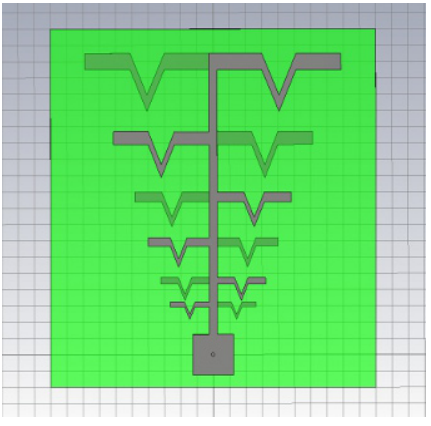

Figure 4. LPKFA with flare angle $45^{\circ}$.

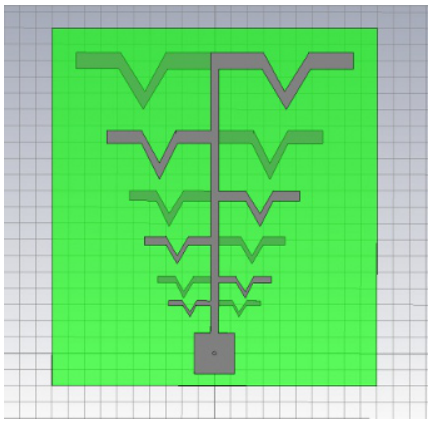

Figure 5. LPKFA with flare angle $60^{\circ}$.

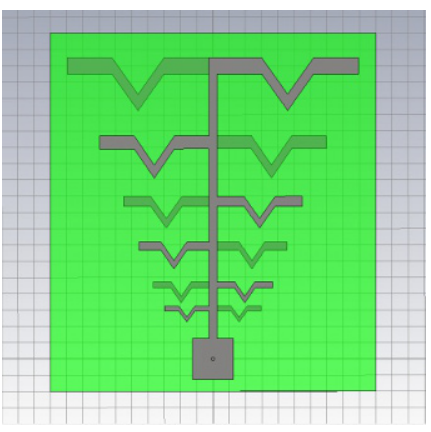

Figure 6. LPKFA with flare angle $70^{\circ}$.

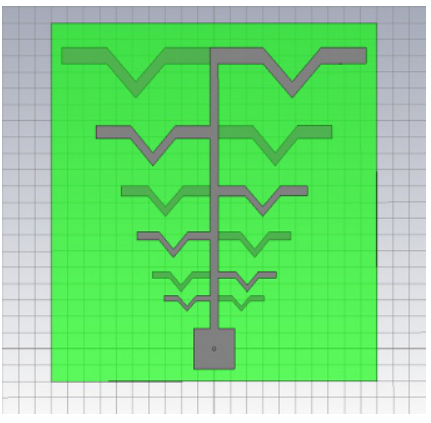

Figure 7. LPKFA with flare angle $80^{\circ}$. 

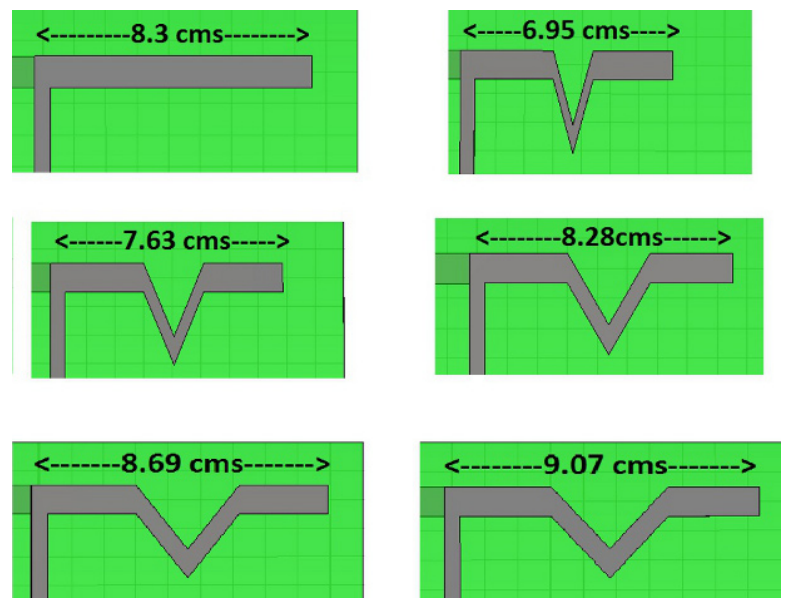

Figure 8. Dimensional difference due to difference in flare angle.

angles at $0^{\circ}, 30^{\circ}, 45^{\circ}, 60^{\circ}, 70^{\circ}$ and $80^{\circ}$. The difference in dimensions caused by the different flare angles are shown in the order of the flare angles $0^{\circ}, 30^{\circ}, 45^{\circ}, 60^{\circ}$, $70^{\circ}$ and $80^{\circ}$. Figure 8 shows dimensional differences due to difference in flare angles of fractal antennas.

As seen in the above Figure 8 , there is a reduction in size of the antenna as angle goes below $60^{\circ}$. This reduction can also be used in the miniaturization of the antenna size.

\section{Simulation results}

The simulation of the above structures have yielded a great number of results showing the far field radiation pattern, gain (in $\mathrm{dB}$ ), directivity (in $\mathrm{dBi}$ ), return loss parameter $\left(S_{11}\right)$ and VSWR (voltage standing wave ratio). The simulation is run on CST - Microwave Studio ${ }^{\circledR}$ using the Transient analysis method at various frequencies ranging from $0.4 \mathrm{GHz}$ to $2.9 \mathrm{GHz}$. The following radiation patterns have been taken for the frequency at $1.37 \mathrm{GHz}$ for the different designed antennas. Figures 9, 10, 11, 12, 13 and 14 show the radiation patterns for flare angles $0,30,45$, 60,70 and 80 degrees respectively.

Figure 9 shows the radiation pattern of normal log periodic antenna at $1.37 \mathrm{GHz}$. Having a maximum gain of $5.6 \mathrm{~dB}$, the radiation pattern is very poor in terms of directivity and the direction of main lobe. As this is an end-fire radiation antenna, the main lobe (maximum gain) should be towards the smaller elements (toward bottom of the diagram). But this is not the case. Hence, it shows poor radiation characteristics.

Figure 10 shows the radiation pattern of the LPKFA with $30^{\circ}$ flare angle at $1.37 \mathrm{GHz}$. It is clear from the figure that the main lobe is towards the smaller elements with

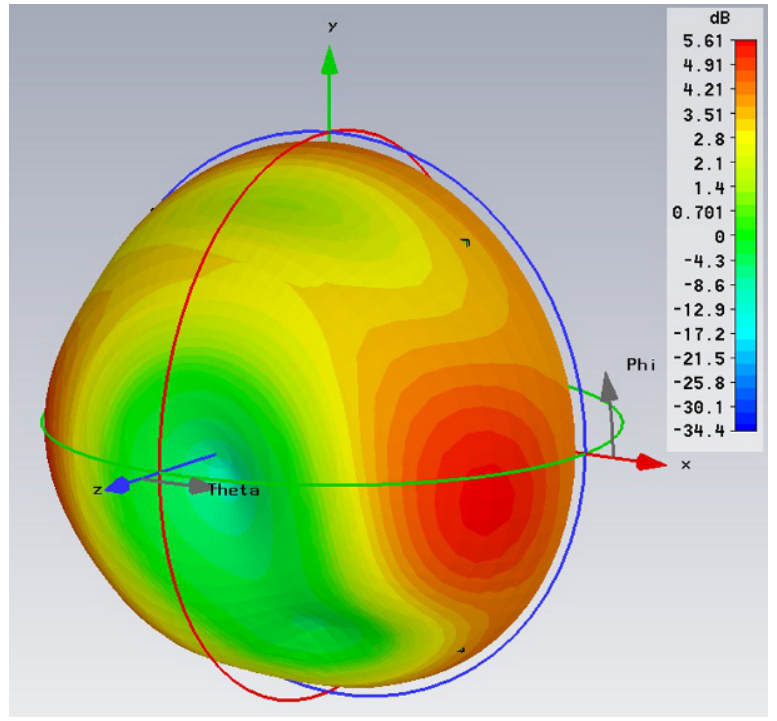

Figure 9. Radiation pattern for LPA.

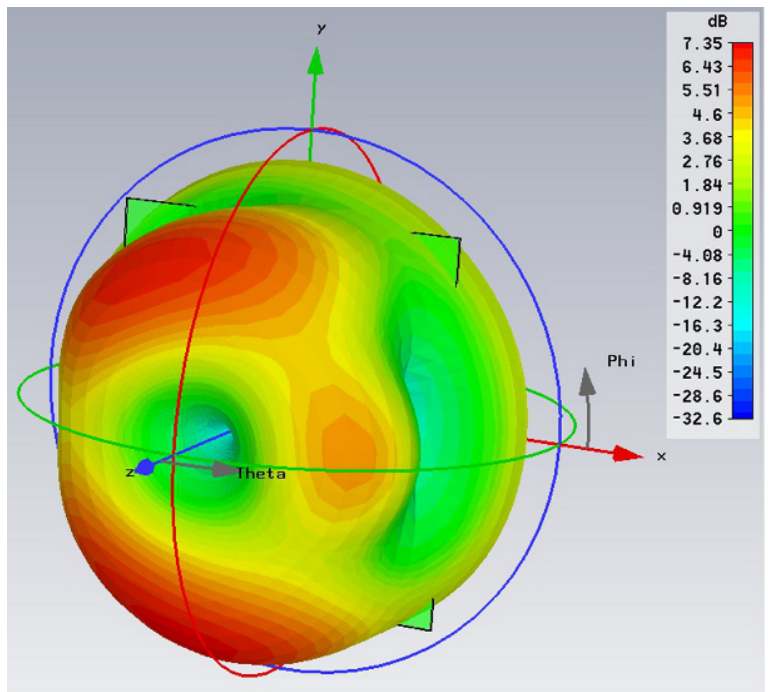

Figure 10. Radiation pattern for $30^{\circ}$.

maximum gain of $7.35 \mathrm{~dB}$. But it is also seen from the figure that there are too many side lobes and the back lobe is too great in magnitude. This also results in unwanted radiation and thus a very poor radiation.

Figure 11 shows the radiation pattern of LPKFA with $45^{\circ}$ flare angle at $1.37 \mathrm{GHz}$. As seen in figure, even though there is an increase in the width of the main lobe (with maximum gain of $7.47 \mathrm{~dB}$ ) in comparison to Figure 10, there is still the presence of large unwanted side lobe radiations.

Figure 12 shows the radiation pattern of LPKFA with flare angle $60^{\circ}$ at $1.37 \mathrm{GHz}$. It can be seen from the figure that 


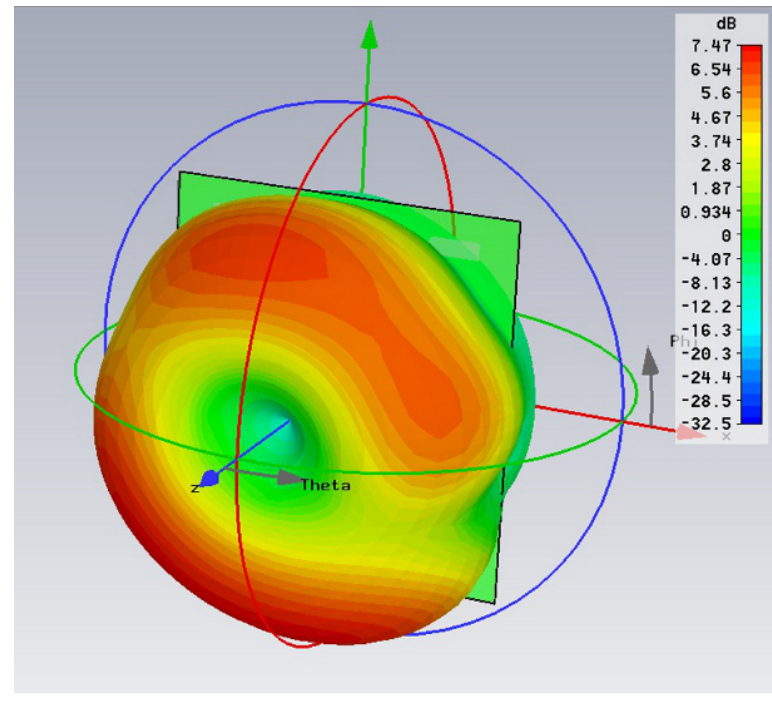

Figure 11. Radiation pattern for $45^{\circ}$.

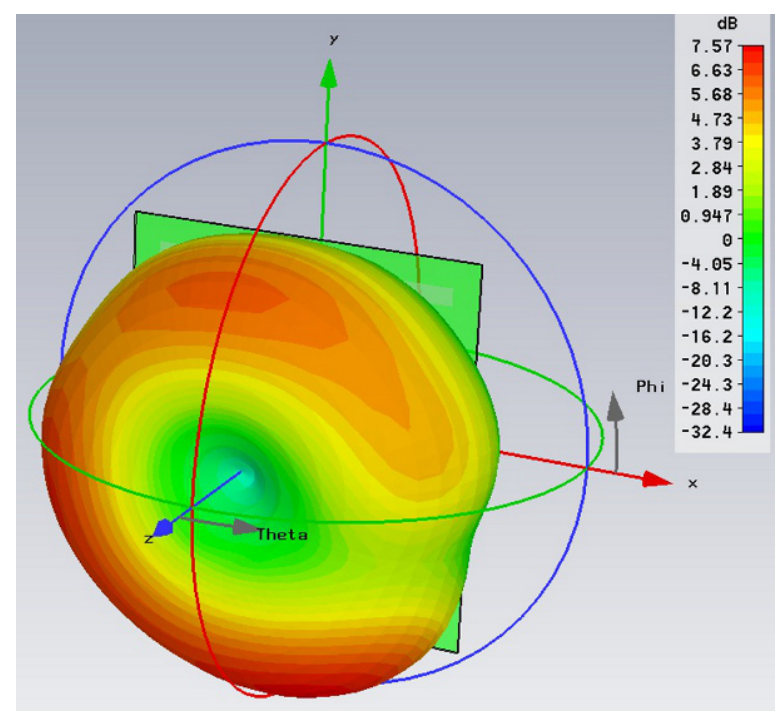

Figure 12. Radiation pattern for $60^{\circ}$.

there is a major increase in the width of the main lobe towards the smaller elements and also optimal back lobe magnitude with increase in main lobe gain magnitude to $7.57 \mathrm{~dB}$.

Figure 13 shows the radiation pattern of LPKFA with $70^{\circ}$ flare angle at $1.37 \mathrm{GHz}$. It is seen that there is a decrease in the main lobe magnitude (maximum gain of $7.23 \mathrm{~dB}$ ) in comparison to Figure 12 with almost no change in the back lobe magnitude.

Figure 14 shows the radiation pattern of LPKFA with $80^{\circ}$ flare angle at $1.37 \mathrm{GHz}$. This pattern also shows a further reduction in the main lobe magnitude in comparison to

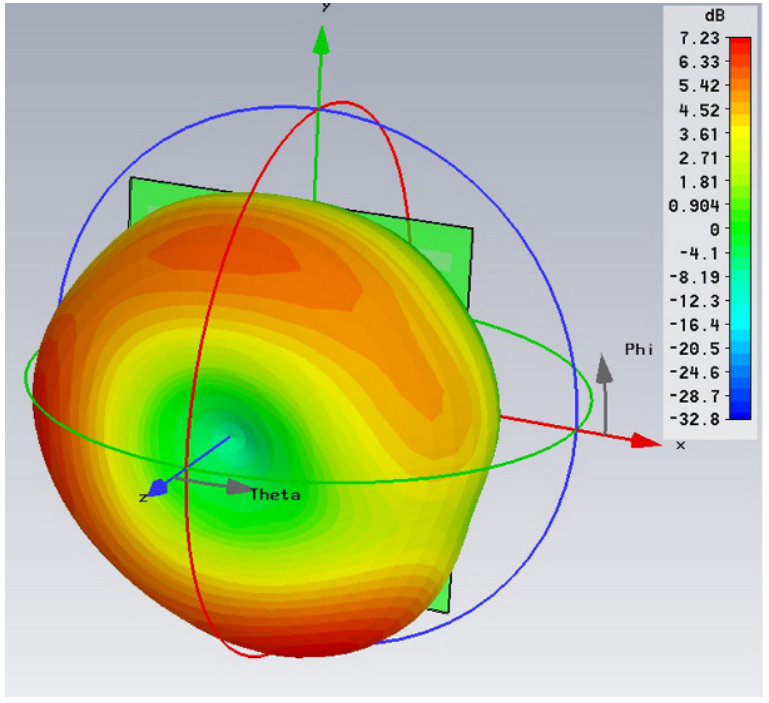

Figure 13. Radiation pattern for $70^{\circ}$.

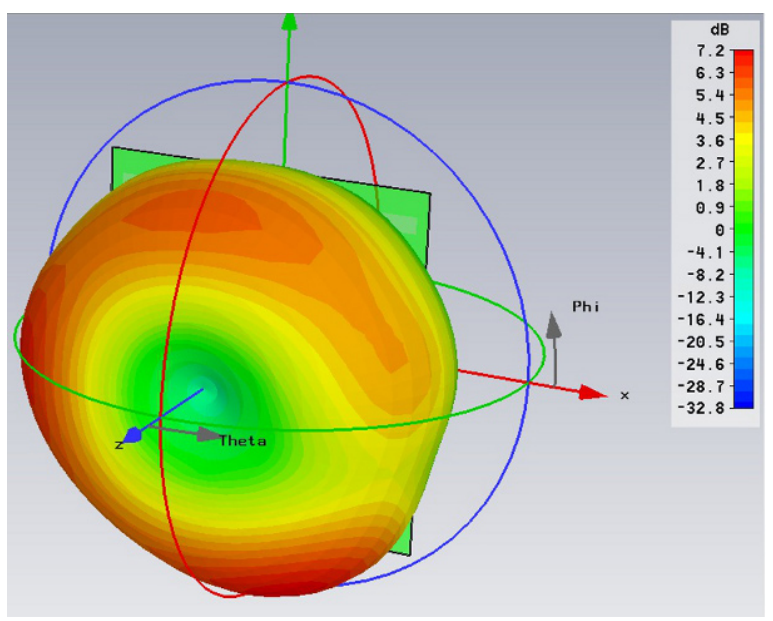

Figure 14. Radiation pattern for $80^{\circ}$.

Figure 12 and 13 with almost no change in the back lobe magnitude.

From the above patterns of Figure 9-14, it can be observed that the antenna with $60^{\circ}$ flare angle provide the best radiation pattern with a maximum gain of $7.575 \mathrm{~dB}$.

The above table. 1 of readings show that the antenna with the flare angle $60^{\circ}$ give the best possible radiation pattern while keeping the size of the antenna almost constant. Figure 15 shows variation of gain with different flare angles. We find that maximum gain is obtained near $60^{\circ}$ flare angle. Similarly Figure 16 shows variation of directivity with different flare angles. In Figure 17 variation of gain is shown for different frequencies. 


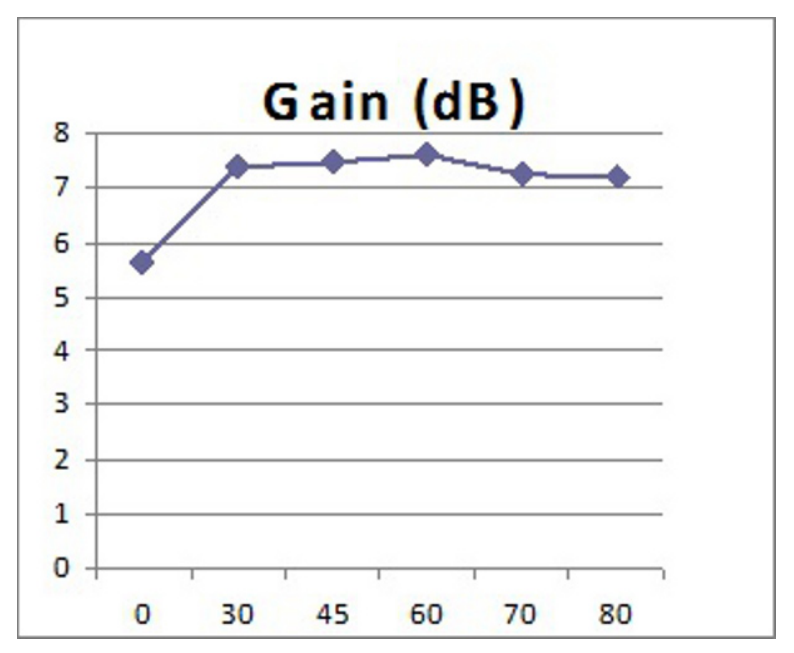

Figure 15. Chart showing variation in Gain vs. Flare angle.

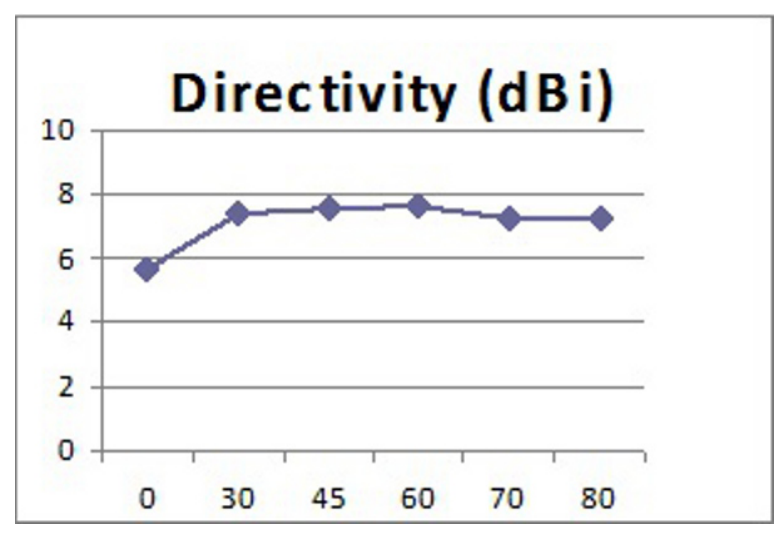

Figure 16. Chart showing variation in Directivity (dBi) vs. Flare angle (degrees).

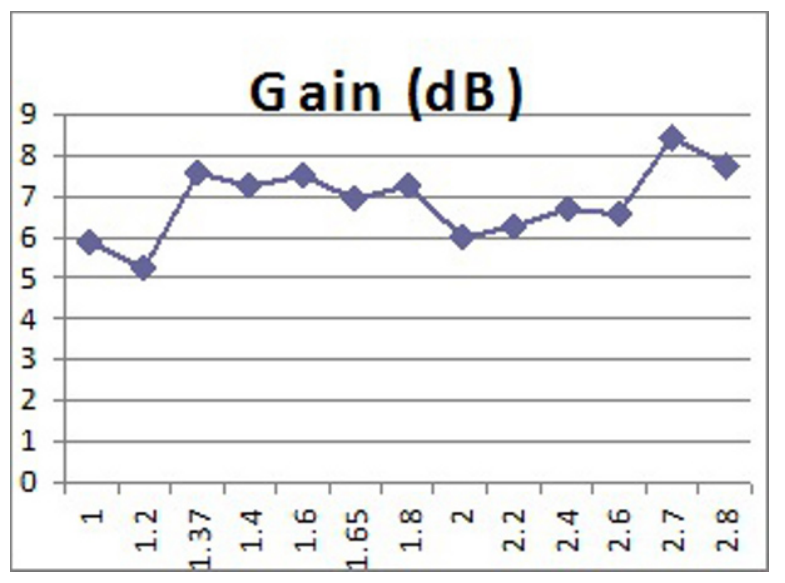

Figure 17. Chart showing variation in Gain $(\mathrm{dB})$ vs. Frequency $(\mathrm{GHz})$ for $60^{\circ}$ flare angle antenna.
Table 1. Simulated gain and directivity at $1.37 \mathrm{GHz}$.

\begin{tabular}{ccc}
\hline $\begin{array}{c}\text { Flare Angle } \\
\text { (degrees) }\end{array}$ & $\begin{array}{c}\text { Gain } \\
(\mathrm{dB})\end{array}$ & $\begin{array}{c}\text { Directivity } \\
(\mathrm{dBi})\end{array}$ \\
\hline \hline LPA & 5.609 & 5.671 \\
30 & 7.352 & 7.420 \\
45 & 7.472 & 7.525 \\
60 & 7.575 & 7.623 \\
70 & 7.230 & 7.276 \\
80 & 7.199 & 7.249 \\
\hline
\end{tabular}

This antenna with $60^{\circ}$ provides a very good radiation pattern with very minor difference in the return loss parameter as compared to the conventional log periodic antenna. The Figure 17 shows the gain variation of the antenna with $60^{\circ}$ flare angle at various frequencies. The simulated return loss parameter of the antenna, with $60^{\circ}$ flare angle, is compared to the conventional $\log$ periodic antenna.

The Figure 18 shows the $S_{11}$ parameter of the antennas with $S_{11}$ along $y$-axis ranging from $-35 \mathrm{~dB}$ to $0 \mathrm{~dB}$ and frequency in $\mathrm{GHz}$ along x-axis ranging from $0-3 \mathrm{GHz}$.

As seen in Figure 18, there is only a slight variation in return loss parameter between the fractal antenna and the conventional log periodic antenna. As seen, the bands of frequencies providing more negative S-parameter (in dB) have been improved too.

The Figure 19 shows the VSWR parameter of the $60^{\circ}$ flare angled LPKFA with VSWR along $y$-axis ranging from 0 to 120 and frequency in $\mathrm{GHz}$ along $x$-axis ranging from 0 to $3 \mathrm{GHz}$.

The graph in Figure 19 shows that this log periodic Koch fractal antenna with $60^{\circ}$ flare angle provides a VSWR $=$ 1.3711695 at $1.37 \mathrm{GHz}$ and VSWR (minimum) $=1.0698082$ at $0.425 \mathrm{GHz}$. The simulated Quality Factor of this antenna, $\mathrm{Q}=5332.1$.

\section{Conclusion}

In this paper, we used the CST - Microwave Studio ${ }^{\circledR}$ to design and simulate the log periodic Koch fractal antenna at various flare angles along with the traditional log periodic antenna. We find that the Koch Fractal antenna provides a better performance than the normal antenna and also that the antenna with a flare angle of $60^{\circ}$ gives optimum performance in terms of radiation pattern, gain and directivity. This $60^{\circ}$ flare angled Koch fractal antenna also provides a return loss parameter of up to $-29 \mathrm{~dB}$ and a VSWR $<2$, which gives very high radiation efficiency. 


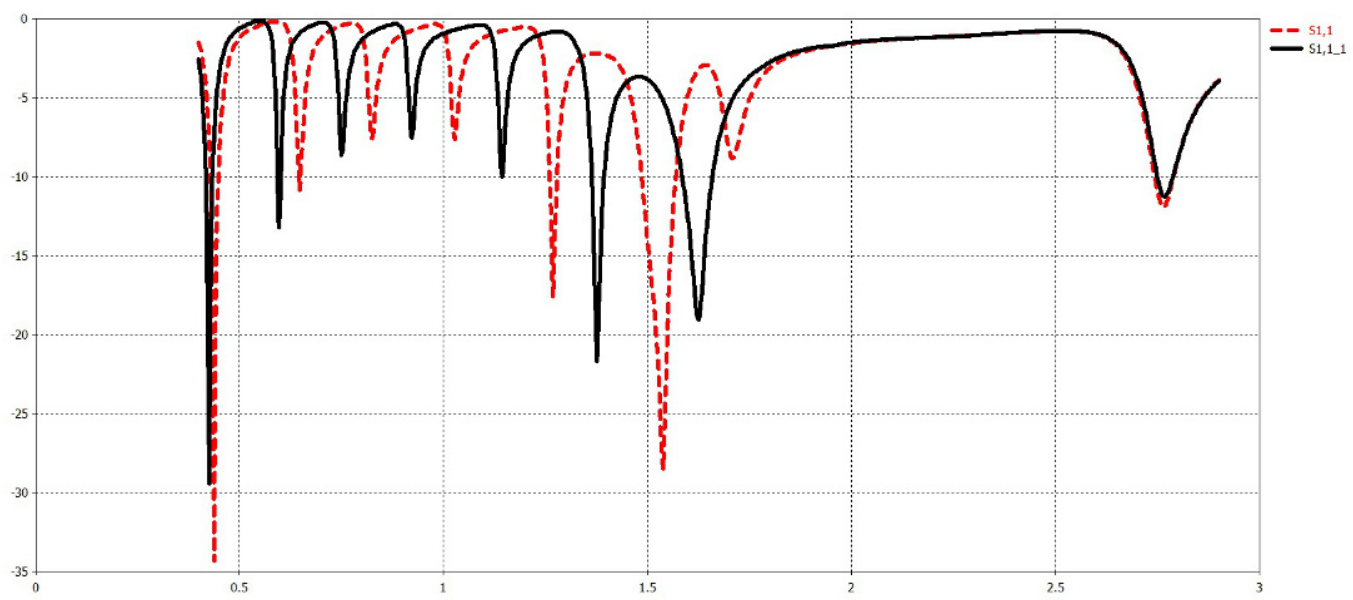

Figure 18. S-parameter (dB) plots of log periodic antenna (dotted line) and log periodic Koch fractal with $60^{\circ}$ flare angle Vs. Frequency $(\mathrm{GHz})$.

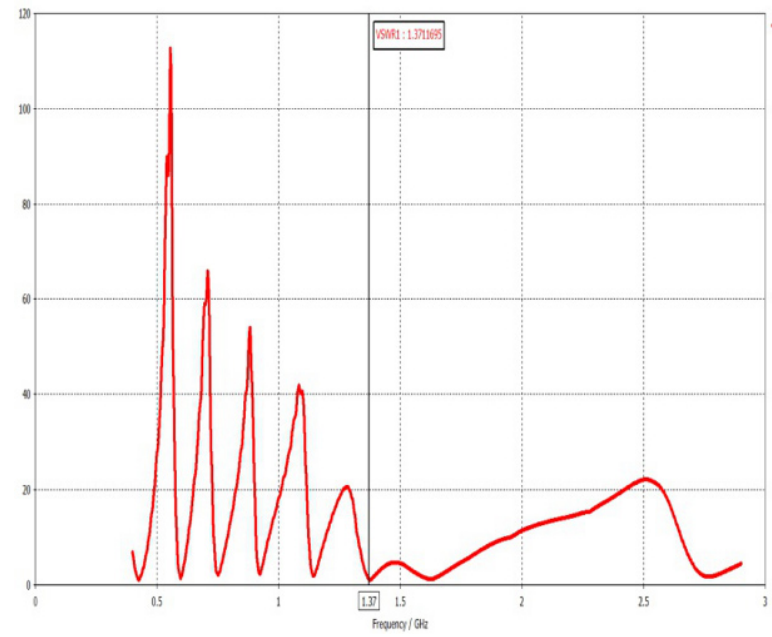

Figure 19. VSWR of Log Periodic Koch Antenna with $60^{\circ}$ flare angle Vs. Frequency $(\mathrm{GHz})$.

\section{References}

[1] Anagnostou D.E., Papapolymerou J., Tentzeris M.M., Christodoulou C.G., A printed log-periodic koch-dipole array (LPKDA), IEEE Antennas and Wireless Propagation Letters, 7, 2008, 456-460

[2] Sharma S.K., Shafai L., Investigations on miniaturized endfire vertically polarized quasi-fractal log-periodic zigzag antenna, IEEE Transactions on Antennas and Propagation, 52 (8), 2004, 1957-1962

[3] Song C.T.P., Hall P.S., Ghafouri-Shiraz H., Shorted fractal Sierpinski monopole antenna, IEEE Transac- tions on Antennas and Propagation, 52 (10), 2004, 2564-2570

[4] Naghshvarian-Jahromi M., Novel Wideband Planar Fractal Monopole Antenna, IEEE Transactions on Antennas and Propagation, 56 (12), 2008, 3844-3849

[5] Strycek M., Hertl I., Fractal log-periodic Antenna, In: IEEE proc., 2007, 1-3

[6] Wang B., Chen A., Su D., An improved fractal tree log-periodic dipole antenna, In: IEEE proc., 2008, 831-834

[7] Qiu J., Lin S., Yang C., You Q., A novel printed fractal log-periodic dipole antenna, In: IEEE proc., 2005, 50-53

[8] Tang P., Scaling property of the Koch fractal dipole, In: IEEE proc., 2001, 3, 150-153,

[9] Moallemizadeh A., Hassani H.R., Nezhad S.M.A., Wide bandwidth and small size LPDA antenna, In: IEEE proc., 2012, 1-3

[10] Werner D.H., Werner P.L., Ferraro A.J. Frequency independent features of self-similar fractal antennas, In: IEEE proc., 2006 3, 2050-2053

[11] Cohen N., Fractal antenna applications in wireless telecommunications, In: IEEE proc., May 1997, 43-49,

[12] Karim M.N.A., Rahim M.K.A., Majid H.A., Ayop O., et al., Zubir log periodic fractal Koch antenna for UHF band applications, PIER 100, 2010, 201-218

[13] Li D.T., Mao J.F., Koch-like sided Serpiski gasket mutifractal dipole antenna, PIER, 126, 2012, 399-427 